\title{
Definitive Pelvic Radiotherapy and Survival of Patients With Newly Diagnosed Metastatic Anal Cancer
}

\author{
Yuefeng Wang, MD, PhDa; Xinhua Yu, PhDº; Nan Zhao, MDc; Jiajing Wang, MSº; Chi Lin, MD, PhDc; \\ Enrique W. Izaguirre, PhD; ; Michael Farmer, MDa; Gary Tian, MD, PhDd; Bradley Somer, MDd; \\ Nilesh Dubal, MDa; David L. Schwartz, MDa; Matthew T. Ballo, MDa; and Noam A. VanderWalde, MD ${ }^{a}$
}

\begin{abstract}
Background: Chemotherapy with or without pelvic radiotherapy (RT) is included in the NCCN Clinical Practice Guidelines in Oncology (NCCN Guidelines) for metastatic anal cancer (MAC), despite limited clinical evidence for RT in this setting. In addition, increasing evidence shows that local therapies, including RT, may increase patient survival for some types of metastatic cancers. The purpose of this study was to evaluate the patterns of care and association between definitive pelvic RT and overall survival (OS) for patients with MAC. Methods: The National Cancer Database was analyzed to evaluate OS of patients with newly diagnosed MAC treated with chemotherapy with or without pelvic RT. Those who did not undergo treatment, treated with surgery, or without baseline variables were excluded. OS was analyzed using the Kaplan-Meier method, log-rank test, Cox proportional hazards models, and propensity score-matched analyses. Results: From 2004 through 2015, 437 patients received chemotherapy alone and 1,020 received pelvic chemoradiotherapy (CRT). At a median follow-up of 17.3 months, CRT was associated with improved $O S$ on univariate $(P<.001)$ and multivariate analysis (hazard ratio $[\mathrm{HR}], 0.70 ; 95 \% \mathrm{Cl}, 0.61-0.81 ; P<.001$ ). Propensity score-matched analysis demonstrated superior median survival (21.3 vs 15.9 months) and 2 -year OS rates (46\% vs 34\%) with CRT compared with chemotherapy alone $(P<.001)$. Landmark analyses limited to long-term survivors of $\geq 1, \geq 2$, and $\geq 4$ years showed improved OS with CRT in all subsets (all $P<.05$ ). CRT with therapeutic doses ( $\geq 45 \mathrm{~Gy}$ ) was associated with longer median survival than palliative doses ( $<45 \mathrm{~Gy}$ ) and chemotherapy alone (24.9 vs 10.9 vs 15.6 months, respectively; $P<.001)$. The benefit of $C R T$ was present among not only those with distant lymph node metastasis ( $H R, 0.63$; $P=.04)$ but also those with distant organ disease (HR, 0.74; $P<.001)$. Conclusions: In this large hypothesis-generating analysis, patients with newly diagnosed MAC who received definitive pelvic RT with chemotherapy lived significantly longer than those who received chemotherapy alone. Prospective trials evaluating definitive local RT for MAC are warranted.
\end{abstract}

J Natl Compr Canc Netw 2019;17(1):29-37 doi: $10.6004 /$ jnccn.2018.7085

aDepartment of Radiation Oncology, West Cancer Center, and bepartment of Preventive Medicine, University of Tennessee Health Science Center, Memphis, Tennessee; 'Department of Radiation Oncology, University of Nebraska Medical Center, Omaha, Nebraska; and dDepartment of Hematology/Oncology, West Cancer Center, University of Tennessee Health Science Center, Memphis, Tennessee.
The standard of care for locally advanced anal cancer is definitive pelvic chemoradiotherapy (CRT). ${ }^{1-5}$ In the metastatic setting, chemotherapy (cisplatin/5-FU, carboplatin/paclitaxel, or FOLFOX) is considered the standard of care, and the role of definitive pelvic radiotherapy (RT) has not been established. ${ }^{6,7}$ However, chemotherapy with or without pelvic RT has been recommended in the NCCN Clinical Practice Guidelines in Oncology (NCCN Guidelines) for metastatic anal cancer (MAC), despite limited clinical evidence for RT in this setting. ${ }^{8}$ Yet, there is increasing evidence showing that local therapies may increase survival for some types of metastatic cancers. ${ }^{9-19}$

Because the literature on the potential benefit of definitive RT to the primary tumor in MAC is unclear, we analyzed the National Cancer Database (NCDB) to assess patterns of care and survival outcomes of chemotherapy with or without RT in patients with MAC. We hypothesized that patients who received definitive doses of pelvic RT for MAC would have improved survival compared with those who did not.

\section{Methods}

\section{Data Source}

The NCDB is a hospital-based cancer registry maintained by both the Commission on Cancer of the American College of Surgeons and the American Cancer Society. ${ }^{20}$ It includes approximately $70 \%$ of cancers diagnosed in the United States. The NCDB was used to retrospectively assess the patterns of care for patients with newly diagnosed MAC from 2004 through 2015. Survival was compared between patients who received pelvic CRT and those who received chemotherapy alone.

\section{Covariates, Treatments, and Patient Characteristics} Eligibility criteria included patients aged $\geq 18$ years with newly diagnosed, histologically confirmed, metastatic

\section{See JNCCN.org for supplemental online content.}


squamous cell carcinoma of the anus treated with upfront chemotherapy (supplemental eFigure 1, available with this article at JNCCN.org). Patients who received no treatments, whose treatment was unknown, who received RT alone or surgery, and who were missing baseline variables or follow-up information were excluded. Patients receiving external-beam RT to the pelvis were considered to be in the CRT group, although those who received brachytherapy were excluded. To minimize the heterogeneity of therapies, patients who received RT to distant nonprimary sites were excluded. Treatment coding in the NCDB is limited to the first course of treatment, defined as all methods of therapy recorded in the treatment plan and administered to the patient before disease progression or recurrence. Data regarding specific agents of chemotherapy administered are not available in the NCDB.

\section{Statistical Analyses}

The primary objective of analysis was the comparison of overall survival (OS) for MAC treated with chemotherapy with and without pelvic RT. OS was estimated using the Kaplan-Meier method, and univariate comparisons were performed using the log-rank test and unadjusted Cox models. Multivariate Cox models were adjusted for factors including RT, age, year of diagnosis, race, comorbidity score, tumor grade, clinical tumor (T) stage, clinical nodal $(\mathrm{N})$ stage, treating facility, insurance status, and metastatic site (distant lymph node, distant organ, or both). Proportional hazards assumption was assessed for all covariates and returned no significant results. Median follow-up was evaluated using the reverse Kaplan-Meier method. Baseline characteristics were compared using the chi-square test for categorical variables; the Mann-Whitney U and Kruskal-Wallis nonparametric tests were used for non-normally distributed variables of 2 and $>2$ groups, respectively.

Propensity score-matched (PSM) analyses were performed comparing outcomes with chemotherapy alone versus CRT. ${ }^{21}$ One-to-one matching without replacement was completed using the nearest-neighbor match on the logit of the propensity score for RT administration (supplemental eTable 1). The caliper width was 0.05 times the standard deviation of the logit of the propensity score, which is estimated to eliminate $>99 \%$ of the bias due to known confounding variables.

To account for potential bias favoring administration of pelvic RT to patients with more favorable baseline prognoses (immortal time bias), sequential landmark analyses evaluating survival with and without pelvic RT were performed for patients surviving a minimum of $\geq 1$, $\geq 2$, and $\geq 4$ years from diagnosis. ${ }^{22}$ Subgroup multivariate Cox regression analyses evaluating the impact of pelvic RT were performed for 6 covariates, including age, race, comorbidity score, $\mathrm{T}$ stage, $\mathrm{N}$ stage, and metastatic site. To further test the role of pelvic RT on patient survival, secondary analysis was performed based on RT dose (total dose $\geq 45$ vs $<45$ Gy).

To rule out the selection bias in which RT was selectively delivered to patients who received chemotherapy first and had good response, we investigated patients who received RT by CRT sequence (concurrent vs nonconcurrent CRT; see definitions in supplemental eAppendix 1). All statistical analyses were performed using SAS 9.4 (SAS Institute).

\section{Results}

\section{Patient Characteristics}

A total of 1,457 patients met our inclusion criteria. Table 1 demonstrates the baseline treatment characteristics between those who received chemotherapy alone and those who received pelvic CRT. More patients received pelvic CRT $(\mathrm{n}=1,020 ; 70 \%)$ than chemotherapy alone $(\mathrm{n}=437 ; 30 \%)$. Patient characteristics were well-balanced for age, race, and Charlson/Deyo comorbidity score between the groups. The use of CRT was associated with earlier year of diagnosis, female sex, higher $\mathrm{T}$ and $\mathrm{N}$ stage, metastases to distant lymph node only, community treatment facility, and nonprivate insurance (Table 1).

\section{Survival Analysis}

Median and mean follow-ups were 17.3 and 25.1 months, respectively. On univariate analysis, CRT was associated with a longer median OS (21.2 vs 15.6 months) and improved 2-year OS (46\% vs $34 \%$ ) and 5-year OS rates (26\% vs 12\%; hazard ratio [HR], 0.69; 95\% CI, 0.61-0.79; $P<.001$; Figure 1A) compared with chemotherapy alone. On multivariate analysis, CRT remained independently associated with improved OS (HR, 0.70; 95\% CI, 0.61$0.81 ; P<.001$; Table 2).

A propensity score analysis was performed, and 406 patients were matched in each treatment group. Patient characteristics were well-balanced across all known covariates (supplemental eTable 1). This matched analysis redemonstrated an association between pelvic CRT and improvements in median survival (21.3 vs 15.9 months), 2 -year OS rate $(46 \%$ vs $34 \%)$, and 5 -year OS rate (26\% vs $12 \%$; HR, $0.67 ; 95 \%$ CI, 0.57-0.79; $P<.001$; Figure 1B).

\section{Landmark Analyses}

Sequential landmark analyses to account for potential bias favoring the administration of pelvic RT to patients with more favorable baseline prognoses showed an association between pelvic RT and improved OS in both univariate and multivariate models for long-term sur- 


\begin{tabular}{|c|c|c|c|c|}
\hline & $\mathbf{N}$ & $\begin{array}{c}\text { RT + Chemo } \\
\text { n (\%) }\end{array}$ & $\begin{array}{c}\text { Chemo Alone } \\
\mathrm{n}(\%)\end{array}$ & $P$ Value $^{a}$ \\
\hline All patients & 1,457 & $1,020(70)$ & $437(30)$ & \\
\hline Year & & & & $<.001$ \\
\hline $2004-2009$ & 589 & $450(44)$ & $139(32)$ & \\
\hline 2010-2015 & 868 & $570(56)$ & $298(68)$ & \\
\hline Sex & & & & .005 \\
\hline Male & 519 & $340(33)$ & $179(41)$ & \\
\hline Female & 938 & $680(67)$ & $258(59)$ & \\
\hline $\mathrm{Age}^{\mathrm{b}} \mathrm{y}$ & & & & .26 \\
\hline$<40$ & 64 & $41(4)$ & $23(5)$ & \\
\hline $40-59$ & 701 & $481(47)$ & $220(50)$ & \\
\hline $60-79$ & 610 & $435(43)$ & $175(40)$ & \\
\hline$\geq 80$ & 82 & $63(6)$ & $19(5)$ & \\
\hline Race & & & & .46 \\
\hline White & 1,240 & $864(85)$ & $376(86)$ & \\
\hline Black & 177 & $130(13)$ & $47(11)$ & \\
\hline Other/Unreported & 40 & $26(2)$ & $14(3)$ & \\
\hline Charlson/Deyo comorbidity score & & & & .32 \\
\hline$\underline{0}$ & 1,192 & $844(83)$ & $348(80)$ & \\
\hline 1 & 179 & $117(11)$ & $62(14)$ & \\
\hline$\geq 2$ & 86 & $59(6)$ & $27(6)$ & \\
\hline T stage & & & & $<.001$ \\
\hline $\mathrm{T1}$ & 54 & $34(3)$ & $20(5)$ & \\
\hline $\mathrm{T} 2$ & 326 & $240(24)$ & $86(20)$ & \\
\hline T3 & 366 & $302(30)$ & $64(15)$ & \\
\hline $\mathrm{T} 4$ & 313 & $230(23)$ & $83(19)$ & \\
\hline$T x$ & 398 & $214(21)$ & $184(42)$ & \\
\hline $\mathrm{N}$ stage & & & & $<.001$ \\
\hline NO & 309 & $208(20)$ & $101(23)$ & \\
\hline N1 & 235 & $161(16)$ & $74(17)$ & \\
\hline $\mathrm{N} 2$ & 278 & $204(20)$ & $74(17)$ & \\
\hline N3 & 406 & $309(30)$ & $97(22)$ & \\
\hline $\mathrm{Nx}$ & 229 & $138(14)$ & $91(21)$ & \\
\hline Metastatic site & & & & $<.001$ \\
\hline Distant lymph node & 282 & $239(23)$ & $43(10)$ & \\
\hline Distant organ & 739 & $502(49)$ & $237(54)$ & \\
\hline Both node and organ & 207 & $130(13)$ & $77(17)$ & \\
\hline Unknown & 229 & $149(15)$ & $80(18)$ & \\
\hline Facility & & & & .003 \\
\hline Academic & 635 & $419(41)$ & $216(49)$ & \\
\hline Community & 758 & $560(55)$ & $198(46)$ & \\
\hline Other/Unknown & 64 & $41(4)$ & $23(5)$ & \\
\hline Insurance & & & & .046 \\
\hline Private & 617 & $408(40)$ & $209(47)$ & \\
\hline Medicare & 536 & $128(13)$ & $46(11)$ & \\
\hline Medicaid/Other ${ }^{c}$ & 536 & $392(38)$ & $144(33)$ & \\
\hline Uninsured & 130 & $92(9)$ & $38(9)$ & \\
\hline Status at last follow-up & & & & .002 \\
\hline Alive & 420 & $319(31)$ & $101(23)$ & \\
\hline Deceased & 1,037 & 701 (69) & $336(77)$ & \\
\hline
\end{tabular}

Abbreviations: Chemo, chemotherapy; Nx, N stage unknown; RT, pelvic radiotherapy; Tx, T stage unknown.

${ }^{a}$ All $P$ values correspond to the chi-square test.

${ }^{\mathrm{b}} \mathrm{Age}$ is coded as 0 to $\geq 90$ years in the National Cancer Database.

Other government insurance. 
A

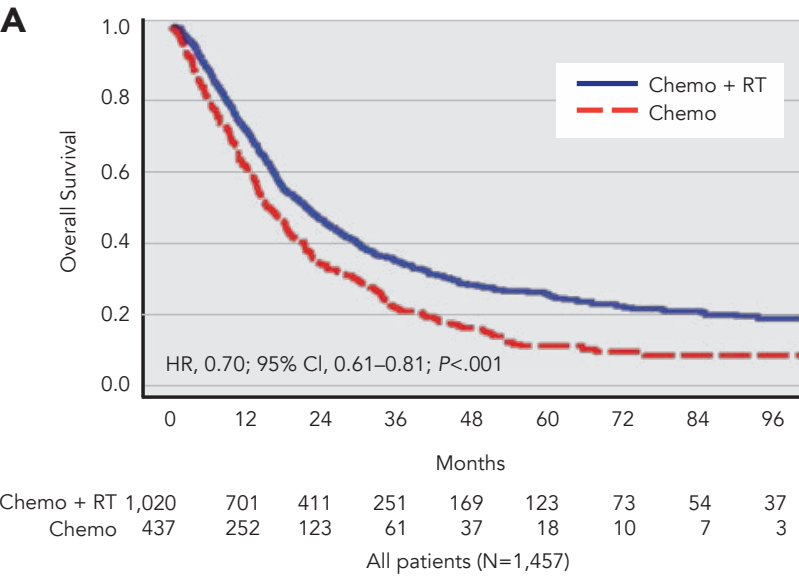

B

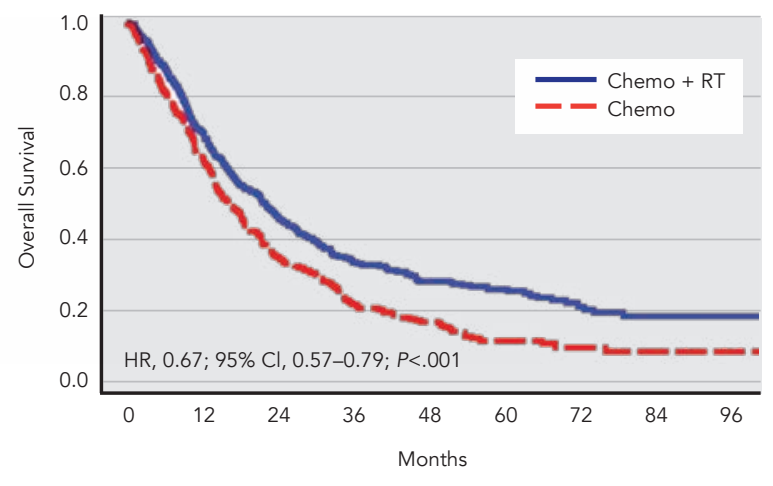

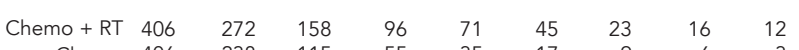
Chemo 406

\begin{tabular}{lccccc}
238 & 115 & 55 & 35 & 17 & 9 \\
\multicolumn{7}{c}{ Propensity } \\
Pcore-matched patients $(\mathrm{N}=812)$
\end{tabular}

Figure 1. Overall survival for (A) all patients and (B) propensity score-matched patients with metastatic anal cancer treated with chemotherapy with or without external-beam RT to the pelvis.

Abbreviations: Chemo, chemotherapy; HR, hazard ratio; RT, pelvic radiotherapy.

vivors of $\geq 1, \geq 2$, and $\geq 4$ years (Figure 2 and supplemental eTable 2; all $P<.05)$. Similar analyses for PSM patients also demonstrated this association at each sequential landmark (supplemental eFigure 2).

\section{Subgroup Analysis}

Subgroup analyses were performed for all patients (Figure 3; supplemental eTable 3). CRT was associated with improved survival compared with chemotherapy alone for all subgroups, including those with distant node-only metastasis (HR, 0.63; 95\% CI, 0.41-0.98; $P=.04$ ) and those with distant organ-only metastasis (HR, 0.74; $95 \%$ CI, 0.61-0.89; $P=.001$ ), but not for patients with both node and organ metastases (HR, 0.92; 95\% CI, 0.63-1.35; $P=.66)$. The magnitude of the association between pelvic RT and improved survival was greater for patients with clinical stage T1-2 tumors (HR, 0.41; 95\% CI, 0.31-0.55; $P<.001$ ) than T3-4 tumors (HR, 0.77; 95\% CI, 0.62-0.95;
$P=.01 ; P$ value for interaction $=.01$ ). No significant interactions were observed between the effects of sex, age, $\mathrm{N}$ status, comorbidity score, and metastatic site.

\section{Definitive Versus Palliative RT}

Patients receiving RT were further stratified into therapeutic versus palliative dose. Among the 1,020 patients who received pelvic RT, 944 had adequate dose information for the analysis. Of those, 755 patients $(80 \%)$ received therapeutic RT (dose $\geq 45$ Gy; median, 54 Gy; range, 45-67.8 Gy;) and 194 (20\%) received palliative RT (dose <45 Gy; median, 32.4 Gy; range, 3.7-43.2 Gy). Compared with palliative RT, therapeutic RT was associated with improved median survival (24.9 vs 10.9 months), 2 -year OS (52\% vs $25 \%)$ and 5 -year OS (29\% vs $14 \%$ ) in both univariate and multivariate analyses (Figure 4A and supplemental eTable 4 ; all $P<.05$ ). The same analysis for PSM patients (Figure 4B and supplemental eTable
A

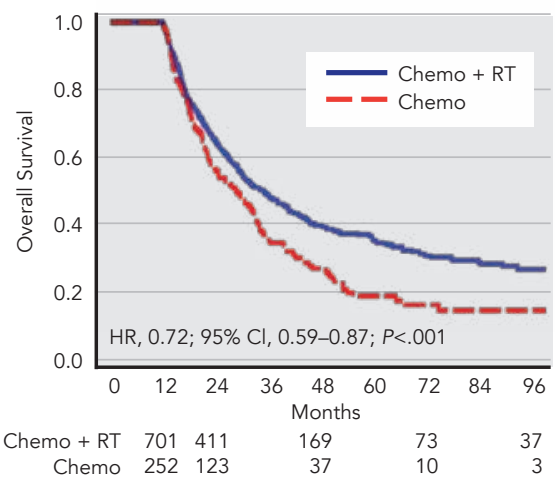

B

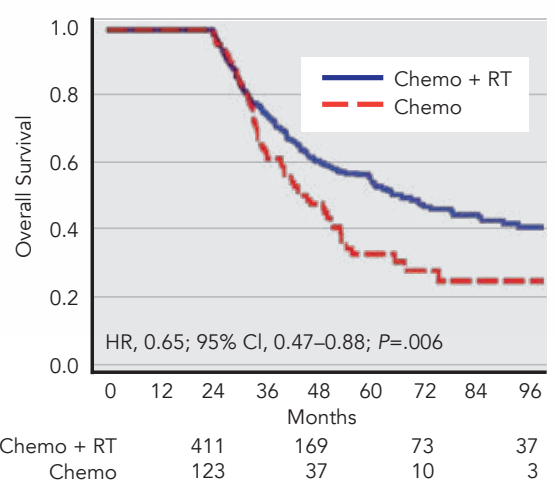

C L Limited to $\geq 4$-Year Survivors

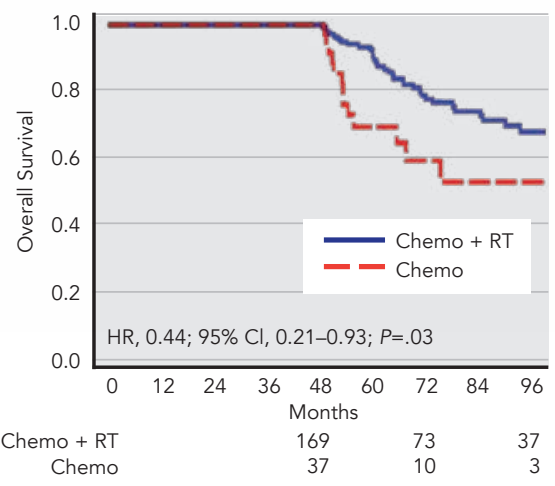

Figure 2. Landmark analyses of overall survival for long-term survivors of $(A) \geq 1,(B) \geq 2$, and $(C) \geq 4$ years.

Abbreviations: Chemo, chemotherapy; HR, hazard ratio; RT, pelvic radiotherapy. 


\begin{tabular}{|c|c|c|c|c|c|c|}
\hline \multirow[b]{2}{*}{ Variable } & \multicolumn{3}{|c|}{ Univariate Analysis } & \multicolumn{3}{|c|}{ Multivariate Analysis } \\
\hline & HR & $95 \% \mathrm{Cl}$ & $P$ Value & $\mathbf{H R}^{\mathbf{a}}$ & $95 \% \mathrm{Cl}$ & $P$ Value \\
\hline \multicolumn{7}{|l|}{ Treatment } \\
\hline Chemo alone & Ref & & & Ref & & \\
\hline RT + Chemo & 0.69 & $0.61-0.79$ & $<.001$ & 0.70 & $0.61-0.81$ & $<.001$ \\
\hline \multicolumn{7}{|l|}{ Year } \\
\hline 2004-2009 & Ref & & & Ref & & \\
\hline 2010-2015 & 0.87 & $0.77-0.99$ & .035 & 0.81 & $0.70-0.93$ & .004 \\
\hline \multicolumn{7}{|l|}{ Race } \\
\hline White & Ref & & & Ref & & \\
\hline Black & 1.01 & $0.83-1.22$ & .92 & 0.84 & $0.67-1.05$ & .12 \\
\hline Other/Unreported & 0.80 & $0.53-1.19$ & .68 & 0.85 & $0.55-1.28$ & .43 \\
\hline \multicolumn{7}{|l|}{ Sex } \\
\hline Male & Ref & & & Ref & & \\
\hline Female & 0.71 & $0.62-0.81$ & $<.001$ & 0.72 & $0.62-0.82$ & $<.001$ \\
\hline \multicolumn{7}{|l|}{ Age, y } \\
\hline$<40$ & Ref & & & Ref & & \\
\hline $40-59$ & 0.48 & $0.35-0.65$ & $<.001$ & 0.75 & $0.55-1.02$ & .06 \\
\hline $60-79$ & 0.60 & $0.45-0.79$ & $<.001$ & 0.94 & $0.69-1.28$ & .69 \\
\hline$\geq 80$ & 1.54 & $1.05-2.27$ & .03 & $\mathrm{~N} / \mathrm{A}$ & $\mathrm{N} / \mathrm{A}$ & N/A \\
\hline \multicolumn{7}{|c|}{ Charlson/Deyo comorbidity score } \\
\hline 0 & Ref & & & Ref & & \\
\hline 1 & 1.42 & $1.17-1.73$ & $<.001$ & 1.43 & $1.17-1.74$ & $<.001$ \\
\hline$\geq 2$ & 1.44 & $1.10-1.89$ & .007 & 1.32 & $1.01-1.74$ & .04 \\
\hline \multicolumn{7}{|l|}{ T stage } \\
\hline $\mathrm{T} 1$ & Ref & & & Ref & & \\
\hline $\mathrm{T} 2$ & 1.44 & $0.94-2.20$ & .09 & 1.29 & $0.84-1.98$ & .24 \\
\hline T3 & 1.73 & $1.13-2.64$ & .01 & 1.55 & $1.01-2.38$ & .04 \\
\hline T4 & 2.07 & $1.36-3.12$ & $<.001$ & 1.82 & $1.19-2.80$ & .005 \\
\hline Tx & 1.89 & $1.24-2.88$ & .002 & 1.55 & $1.02-2.37$ & .04 \\
\hline \multicolumn{7}{|l|}{$\mathrm{N}$ stage } \\
\hline No & Ref & & & Ref & & \\
\hline N1 & 1.06 & $0.85-1.33$ & .58 & 1.11 & $0.88-1.41$ & .37 \\
\hline $\mathrm{N} 2$ & 0.98 & $0.79-1.22$ & .89 & 1.02 & $0.82-1.27$ & .84 \\
\hline N3 & 1.19 & $0.99-1.44$ & .08 & 1.33 & $1.08-1.64$ & .02 \\
\hline $\mathrm{Nx}$ & 1.23 & $0.99-1.53$ & .06 & 1.18 & $0.94-1.48$ & .15 \\
\hline \multicolumn{7}{|l|}{ Facility } \\
\hline Academic & Ref & & & Ref & & \\
\hline Community & 1.19 & $1.03-1.36$ & .01 & 1.16 & $1.01-1.33$ & .03 \\
\hline Other/Unknown & 1.28 & $0.94-1.74$ & .12 & 0.63 & $0.41-0.96$ & .03 \\
\hline \multicolumn{7}{|l|}{ Insurance } \\
\hline Private & Ref & & & Ref & & \\
\hline Medicare & 1.46 & $1.27-1.69$ & $<.001$ & 1.21 & $1.01-1.45$ & .04 \\
\hline Medicaid/Other ${ }^{b}$ & 1.34 & $1.07-1.67$ & .01 & 1.32 & $1.05-1.65$ & .01 \\
\hline Uninsured & 1.58 & $1.24-2.00$ & $<.001$ & 1.44 & $1.12-1.83$ & .003 \\
\hline \multicolumn{7}{|l|}{ Metastatic site } \\
\hline Distant lymph node & Ref & & & Ref & & \\
\hline Distant organ & 1.79 & $1.49-2.14$ & $<.001$ & 1.64 & $1.37-1.98$ & $<.001$ \\
\hline Both node and organ & 2.05 & $1.64-2.56$ & $<.001$ & 1.80 & $1.46-2.31$ & $<.001$ \\
\hline Unknown & 1.66 & $1.33-2.08$ & $<.001$ & 1.63 & $1.29-2.05$ & $<.001$ \\
\hline
\end{tabular}

Abbreviations: Chemo, chemotherapy; HR, hazard ratio; N/A, not available; Nx, N stage unknown; RT, pelvic radiotherapy; Tx, T stage unknown.

aMultivariate HRs are adjusted for all factors shown, as described in supplemental eAppendix 1, available with this article at JNCCN.org.

bOther government insurance. 


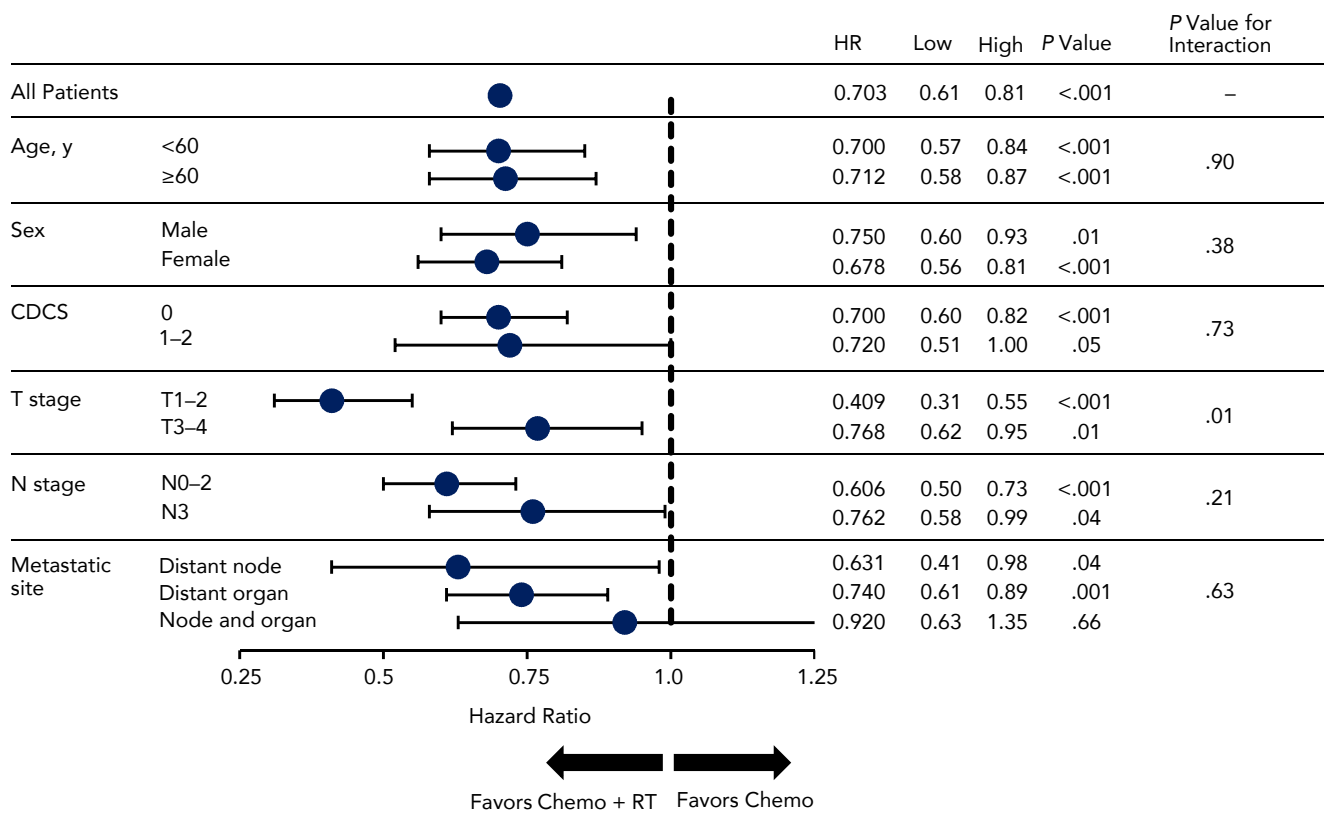

Figure 3. Forest plot of the association between pelvic RT and overall survival by subgroup.

Abbreviations: CDCS, Charlson/Deyo comorbidity score; Chemo, chemotherapy; high, upper limit of the 95\% Cl; HR, multivariate adjusted hazard ratio associated with RT (chemo alone is the reference [HR, 1.0]); low, lower limit of the 95\% Cl; RT, pelvic radiotherapy.

5) showed a similar association with improved median survival (24.6 vs 10.3 months) and 2-year OS rate (51\% vs $26 \%$ ). Patients who received therapeutic RT also had significant longer median survival (24.6 vs 15.9 months; $P<.001)$ compared with those treated with chemotherapy alone. No difference was seen in OS between palliative RT and chemotherapy alone in both univariate (HR, 1.22; 95\% CI, 0.94-1.60; $P=.14$ ) and multivariate analysis (HR, $1.14 ; 95 \%$ CI, 0.86-1.51; $P=.35)$.

\section{Concurrent Versus Nonconcurrent CRT}

Among patients who received RT, 803 (78\%) received concurrent CRT and 217 (22\%) received nonconcurrent CRT. Both concurrent CRT (multivariate HR, 0.69; $P<.001$ ) and nonconcurrent CRT (multivariate HR, $0.74 ; P=.002$ ) had improved median survival compared with chemotherapy alone (Figure 4C and supplemental eTable 6). However, nonconcurrent CRT had slightly worse 2-year OS than concurrent CRT (41\% vs $48 \%$, respectively).

\section{Discussion}

The standard of care for patients with locoregional anal cancer is definitive pelvic CRT with concurrent 5-FU/ mitomycin. ${ }^{2-5}$ In the metastatic setting, however, treatment is not as clear. For decades, chemotherapy (cisplatin/5-FU, carboplatin/paclitaxel, or FOLFOX) was considered the standard of care for MAC. ${ }^{6,7}$ Since 2012, chemotherapy with or without pelvic RT has been in- cluded in the NCCN Guidelines for MAC, despite limited clinical evidence for RT in this setting. ${ }^{8}$ Our study suggests that most patients are receiving pelvic RT even in the setting of metastatic disease.

In this hypothesis-generating analysis, patients with newly diagnosed MAC who received pelvic CRT had improved median, 2-year, and 5-year OS compared with those receiving chemotherapy alone. The benefit of definitive pelvic RT was present among not only those with distant lymph node metastasis (HR, 0.63; $P=.04)$ but also those with distant organ disease (HR, $0.74 ; P<.001)$. The biases in this study were controlled by several methods, including univariate/multivariate analyses and PSM analysis. Disease burden was controlled by $\mathrm{T} / \mathrm{N}$ stage for the primary tumor and by metastatic sites for distant disease (distant node only, distant organ only, or both). To account for potential selection biases between "responder" and "nonresponder" (ie, immortal time bias), sequential landmark analyses showed significant improvement in survival with RT for long-term survivors of $\geq 1, \geq 2$, and $\geq 4$ years, suggesting that the benefit of pelvic RT found in this study is not just due to bias. Lastly, by using the time of treatment initiation (chemotherapy or RT), we found that $78 \%$ of patients treated with CRT received concurrent CRT and had slightly better survival than those treated with nonconcurrent CRT, which helped rule out the selection bias that RT was selectively delivered to patients who received chemotherapy first and had good response. 
A

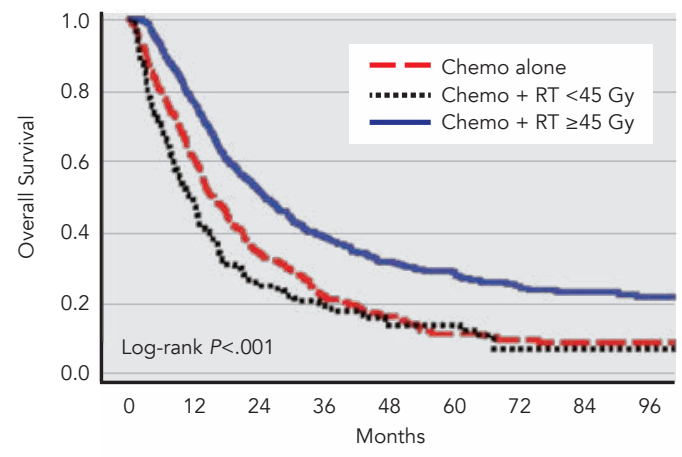

$\begin{array}{llllllllll}\text { Chemo alone } & 437 & 252 & 123 & 61 & 37 & 18 & 10 & 7 & 3\end{array}$ $\begin{array}{lllllllllr}\text { Chemo + RT }<45 \text { Gy } & 194 & 90 & 39 & 27 & 14 & 11 & 4 & 1 & 1\end{array}$ $\begin{array}{llllllllll}\text { Chemo + RT } \geq 45 \text { Gy } & 755 & 558 & 345 & 204 & 138 & 97 & 57 & 44 & 30\end{array}$

B

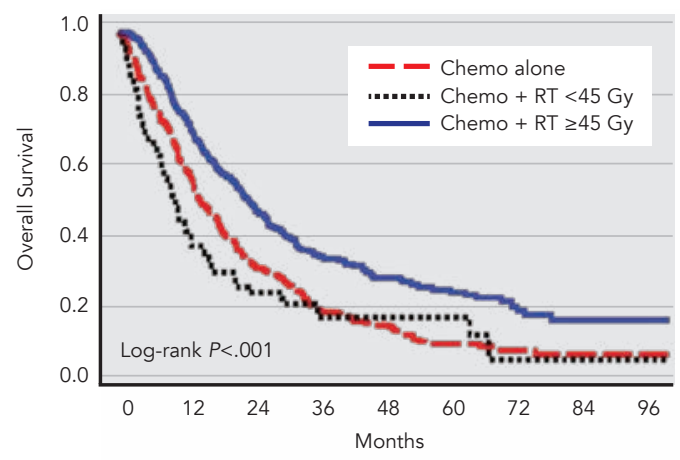

$\begin{array}{llllllllll}\text { Chemo alone } & 406 & 238 & 115 & 55 & 35 & 17 & 9 & 6 & 3\end{array}$

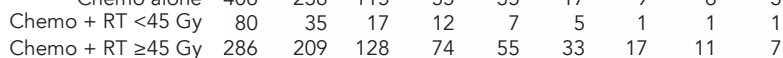

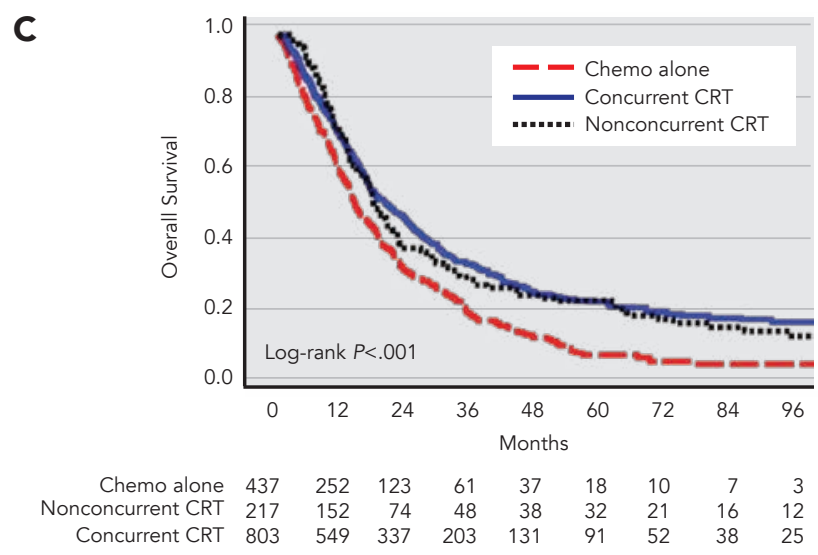

Figure 4. Overall survival analyses by radiation dose $(<45$ and $\geq 45$ Gy) and CRT sequence (concurrent vs nonconcurrent). Multivariate analyses were adjusted for identical factors to the primary analysis, as described in supplemental eAppendix 1. (A) All patients with dose information $(n=1,386)$. (B) Propensity score-matched patients with dose information $(n=772)$. (C) Analysis of all patients by CRT sequence $(n=1,457)$.

Abbreviations: Chemo, chemotherapy; CRT, chemoradiation; HR, hazard ratio; RT, pelvic radiotherapy.
In a separate analysis, patients who received RT treatments to distant nonprimary sites were also included $(n=74)$. Inclusion of these additional patients did not affect our conclusion either in the analysis of all patients and or in the PSM analysis (supplemental eFigure 3).

Data regarding local therapy for MAC are limited in the literature. To our knowledge, this analysis represents the largest reported cohort of patients with MAC treated with external-beam RT, as well as the largest MAC cohort managed with local therapy (RT or surgery) overall. Recently, an analysis using the NCDB for patients diagnosed from 2004 through 2013 showed an association of pelvic RT with improved survival. ${ }^{23}$ However, our analyses used more comprehensive and robust statistical methods, which had better control of biases (univariate/multivariate analyses, PSM, landmark analysis, subgroup analysis, and analysis by sequence of treatments) and provided evidence that definitive pelvic RT was associated with improved OS based on survival analysis stratified by radiation dose (total dose $\geq 45$ vs $<45 \mathrm{~Gy}$ ). In a retrospective study, Eng et $\mathrm{al}^{24}$ showed that survival for 33 patients who underwent multidisciplinary intervention (surgery, RT, or ablation) and chemotherapy was improved compared with patients treated with chemotherapy alone. In another retrospective study, Faivre et $\mathrm{al}^{6}$ found that 10 patients who received local therapy with chemotherapy lived longer than those treated with chemotherapy alone.

How definitive local RT may improve survival for patients with MAC is not clear. There are several possible explanations. First, most patients with MAC would experience locoregional symptoms of pain, bleeding, or obstruction due to locoregional invasion of the tumor. These symptoms could ultimately lead to life-threatening complications, including obstructions and infections that impact life expectancy. Of note, as shown in the survival analysis by dose (Figure 4 and supplemental eTable 4), patients who required palliative RT had worse 2-year OS $(25 \%)$ than those who received chemotherapy alone (34\%), but survival for patients who received definitive RT $(52 \%)$ was much better. Normally, palliative RT is delivered to patients with severe complications, and these patients may have worse disease than those not requiring palliative RT, leading to worse survival compared with those receiving chemotherapy alone. However, upfront definitive pelvic RT could potentially prevent these problems and therefore extend survival.

Second, the seed-and-soil theory provides a biologic rationale for definitive RT of the primary tumor in patients with newly diagnosed MAC. ${ }^{25-27}$ According to this theory, soluble growth factors produced by the primary tumor are required to create a microenvironment that promotes dissemination of malignant clones and formation of metastasis. ${ }^{28,29}$ Consequently, eradication of the primary tumor through definitive RT would decrease the spread of malig- 
nant clones and subsequently decrease the formation of further metastasis. Of note, in subgroup analyses (Figure 3 ), we found that higher T stage (T3-4 vs T1-2) was associated with worse survival. T3-4 tumors had larger primary tumors (vs T1-2) and were less likely to be eradicated by RT, leading to worse survival. This result suggested that control of the primary tumor may play an important role in newly diagnosed MAC.

Third, definitive RT of the primary tumor might have an abscopal effect on metastatic tumors. RT first inflicts damage on cancer cells, eliciting phenotype changes, releasing neoantigens, and subsequently immunizing patients against metastatic cancer by converting the irradiated cancer cells into an in situ vaccine. ${ }^{30,31}$ Golden et al ${ }^{32}$ and Shi et $\mathrm{al}^{33}$ confirmed this immune-mediated response with combination RT and granulocyte macrophage colony-stimulating factor in patients with metastatic nonsmall cell lung, pancreatic, or breast cancer. Further studies of combinations of immune checkpoint inhibitors and RT showed promising therapeutic outcomes in several solid malignancies, including melanoma and non-small cell lung cancer ${ }^{34-37}$ In the future, rational combinations of definitive local RT with immunotherapy, as well as consolidative therapies to metastatic sites, might offer new therapeutic strategies for newly diagnosed MAC. ${ }^{38-40}$

This study has several limitations. Information on specific chemotherapy agents, salvage therapies, and patient HIV and human papillomavirus status (which have been shown to correlate with patient survival ${ }^{41,42}$ ) is not available in the NCDB, and these 4 variables were not controlled in our study. Immortal time bias (ie, patients who died before they could receive RT) and selection bias (ie, RT selectively delivered to patients who received chemotherapy first and had good response) may also impact the results of this study. We tried to account for these potential biases through landmark analyses and survival analysis for concurrent versus nonconcurrent CRT. Although comorbidity was estimated using Charlson/Deyo comorbidity index, that is not a good surrogate for performance status, which is not available in NCDB. Finally, treatment-related toxicity, colostomy-free survival, and disease-specific survival are not coded in NCDB. Despite these limitations, the results of this analysis are intriguing.

\section{Conclusions}

In this large hypothesis-generating analysis, patients with newly diagnosed MAC who received definitive pelvic RT with chemotherapy lived significantly longer than those who received chemotherapy alone. Although optimal patient selection remains uncertain, we observed a benefit across virtually all subgroups of patients. Randomized trials to evaluate the impact of definitive local RT for patients with newly diagnosed MAC appear warranted.

Submitted May 21, 2018; accepted for publication September 12, 2018. Disclosures: Dr. Ballo has disclosed that he is a member of the speakers bureau and serves as a consultant for Novocure. The remaining authors have disclosed that they have not received any financial considerations from any person or organization to support the preparation, analysis, results, or discussion of this article.

Author contributions: Study concept and design: All authors. Data acquisition: All authors. Data analysis and interpretation: All authors. Manuscript preparation: Y. Wang, VanderWalde. Critical revision: All authors. Statistical analysis: Y. Wang, Yu, Zhao, J. Wang, Lin, VanderWalde. Administrative, technical, or material support: All authors. Study supervision: VanderWalde.

Correspondence: Noam A. VanderWalde, MD, Department of Radiation Oncology, West Cancer Center, University of Tennessee Health Science Center, 7945 Wolf River Boulevard, Memphis, TN 38138.

Email: nvanderw@westclinic.com

\section{References}

1. Shridhar R, Shibata D, Chan E, et al. Anal cancer: current standards in care and recent changes in practice. CA Cancer J Clin 2015;65:139-162.

2. Ajani JA, Winter KA, Gunderson LL, et al. Fluorouracil, mitomycin and radiotherapy vs fluorouracil, cisplatin, and radiotherapy for carcinoma of the anal canal: a randomized controlled trial. JAMA 2008;299:1914-1921.

3. James RD, Glynne-Jones R, Meadows HM, et al. Mitomycin or cisplatin chemoradiation with or without maintenance chemotherapy for treatment of squamous-cell carcinoma of the anus (ACT II): a randomised, phase 3, open-label, 2 × 2 factorial trial. Lancet Oncol 2013;14:516-524.

4. Kachnic LA, Winter K, Myerson RJ, et al. RTOG 0529: a phase 2 evaluation of dose-painted intensity modulated radiation therapy in combination with 5-fluorouracil and mitomycin- $\mathrm{C}$ for the reduction of acute morbidity in carcinoma of the anal canal. Int J Radiat Oncol Biol Phys 2013;86:27-33.

5. Holliday EB, Lester SC, Harmsen WS, et al. Extended-field chemoradiation therapy for definitive treatment of anal canal squamous cell carcinoma involving the para-aortic lymph nodes. Int J Radiat Oncol Biol Phys 2018;102:102-108.

6. Faivre C, Rougier P, Ducreux M, et al. [5-fluorouracile and cisplatinum combination chemotherapy for metastatic squamous-cell anal cancer]. Bull Cancer 1999;86:861-865 [in French].

7. Ghosn M, Kourie HR, Abdayem $P$, et al. Anal cancer treatment: current status and future perspectives. World J Gastroenterol 2015;21:22942302.

8. Benson AB III, Venook AP, Al-Hawary MM, et al. Anal Carcinoma, Version 2.2018, NCCN Clinical Practice Guidelines in Oncology. J Natl Compr Canc Netw 2018;16:852-871.

9. Wang $Y$, Farmer $M$, Izaguirre $E$, et al. Association of definitive pelvic radiotherapy and survival for patients with newly diagnosed metastatic cervical cancer. JAMA Oncol 2018;4:1288-1291.

10. Slotman BJ, van Tinteren $\mathrm{H}$, Praag JO, et al. Use of thoracic radiotherapy for extensive stage small-cell lung cancer: a phase 3 randomised controlled trial. Lancet 2015;385:36-42.

11. Mickisch GH, Garin A, van Poppel H, et al. Radical nephrectomy plus interferon-alfa-based immunotherapy compared with interferon alfa alone in metastatic renal-cell carcinoma: a randomised trial. Lancet 2001;358:966-970.

12. Flanigan RC, Salmon SE, Blumenstein BA, et al. Nephrectomy followed by interferon alfa- $2 b$ compared with interferon alfa-2b alone for metastatic renal-cell cancer. N Engl J Med 2001;345:1655-1659.

13. Rusthoven CG, Jones BL, Flaig TW, et al. Improved survival with prostate radiation in addition to androgen deprivation therapy for men with newly diagnosed metastatic prostate cancer. J Clin Oncol 2016;34:2835-2842.

14. Hanna N, Sun M, Meyer CP, et al. Survival analyses of patients with 
metastatic renal cancer treated with targeted therapy with or without cytoreductive nephrectomy: a National Cancer Data Base study. J Clin Oncol 2016;34:3267-3275:

15. Culp SH, Schellhammer PF, Williams MB. Might men diagnosed with metastatic prostate cancer benefit from definitive treatment of the primary tumor? A SEER-based study. Eur Urol 2014;65:1058-1066.

16. Seisen T, Sun M, Leow JJ, et al. Efficacy of high-intensity local treatment for metastatic urothelial carcinoma of the bladder: a propensity score-weighted analysis from the National Cancer Data Base. J Clin Oncol 2016:34:3529-3536.

17. Ost P, Reynders D, Decaestecker K, et al. Surveillance or metastasis-directed therapy for oligometastatic prostate cancer recurrence: a prospective, randomized, multicenter phase II trial. J Clin Oncol 2018;36:446-453.

18. Gomez DR, Blumenschein GR Jr, Lee JJ, et al. Local consolidative therapy versus maintenance therapy or observation for patients with oligometastatic non-small-cell lung cancer without progression after first-line systemic therapy: a multicentre, randomised, controlled, phase 2 study. Lancet Oncol 2016;17:1672-1682.

19. O'Shaughnessy MJ, McBride SM, Vargas HA, et al. A pilot study of a multimodal treatment paradigm to accelerate drug evaluations in early-stage metastatic prostate cancer. Urology 2017;102:164-172.

20. Boffa DJ, Rosen JE, Mallin K, et al. Using the National Cancer Database for outcomes research: a review. JAMA Oncol 2017;3:1722-1728.

21. Guo SY, Fraser MW. Propensity Score Analysis: Statistical Methods and Applications. Los Angeles, CA: SAGE Publications; 2014.

22. Anderson JR, Cain KC, Gelber RD. Analysis of survival by tumor response. J Clin Oncol 1983;1:710-719.

23. Repka MC, Aghdam N, Karlin AW, et al. Social determinants of stage IV anal cancer and the impact of pelvic radiotherapy in the metastatic setting. Cancer Med 2017;6:2497-2506.

24. Eng C, Chang GJ, You YN, et al. The role of systemic chemotherapy and multidisciplinary management in improving the overall survival of patients with metastatic squamous cell carcinoma of the anal canal. Oncotarget 2014;5:11133-11142.

25. Comen E, Norton L, Massague J. Clinical implications of cancer self-seeding. Nat Rev Clin Oncol 2011;8:369-377.

26. Hiratsuka $\mathrm{S}$, Watanabe $\mathrm{A}, \mathrm{Aburatani} \mathrm{H}$, et al. Tumour-mediated upregulation of chemoattractants and recruitment of myeloid cells predetermines lung metastasis. Nat Cell Biol 2006;8:1369-1375.

27. Danna EA, Sinha P, Gilbert M, et al. Surgical removal of primary tumor reverses tumor-induced immunosuppression despite the presence of metastatic disease. Cancer Res 2004;64:2205-2211.

28. Leung CT, Brugge JS. Tumor self-seeding: bidirectional flow of tumor cells. Cell 2009;139:1226-1228.

29. Newton PK, Mason J, Bethel K, et al. Spreaders and sponges define metastasis in lung cancer: a Markov chain Monte Carlo mathematical model. Cancer Res 2013;73:2760-2769.

30. Demaria $\mathrm{S}, \mathrm{Ng}$ B, Devitt ML, et al. lonizing radiation inhibition of distant untreated tumors (abscopal effect) is immune mediated. Int $J$ Radiat Oncol Biol Phys 2004;58:862-870.

31. Vanpouille-Box C, Pilones KA, Wennerberg $E$, et al. In situ vaccination by radiotherapy to improve responses to anti-CTLA-4 treatment. Vaccine 2015;33:7415-7422.

32. Golden EB, Chhabra A, Chachoua A, et al. Local radiotherapy and granulocyte-macrophage colony-stimulating factor to generate abscopal responses in patients with metastatic solid tumours: a proof-of-principle trial. Lancet Oncol 2015;16:795-803.

33. Shi $F$, Wang $X$, Teng $F$, et al. Abscopal effect of metastatic pancreatic cancer after local radiotherapy and granulocyte-macrophage colony-stimulating factor therapy. Cancer Biol Ther 2017;18:137-141.

34. Herrera FG, Bourhis J, Coukos G. Radiotherapy combination opportunities leveraging immunity for the next oncology practice. CA Cancer Clin 2017;67:65-85.

35. Aboudaram A, Modesto A, Chaltiel L, et al. Concurrent radiotherapy for patients with metastatic melanoma and receiving anti-programmed-death 1 therapy: a safe and effective combination. Melanoma Res 2017;27:485-491.

36. Golden EB, Demaria S, Schiff PB, et al. An abscopal response to radiation and ipilimumab in a patient with metastatic non-small cell lung cancer. Cancer Immunol Res 2013;1:365-372.

37. Shaverdian N, Lisberg AE, Bornazyan K, et al. Previous radiotherapy and the clinical activity and toxicity of pembrolizumab in the treatment of non-small-cell lung cancer: a secondary analysis of the KEYNOTE-001 phase 1 trial. Lancet Oncol 2017;18:895-903.

38. Ott PA, Piha-Paul SA, Munster P, et al. Safety and antitumor activity of the anti-PD-1 antibody pembrolizumab in patients with recurrent carcinoma of the anal canal. Ann Oncol 2017;28:1036-1041.

39. Morris VK, Salem ME, Nimeiri H, et al. Nivolumab for previously treated unresectable metastatic anal cancer (NC19673): a multicentre, single-arm, phase 2 study. Lancet Oncol 2017;18:446-453.

40. lyengar P, Wardak Z, Gerber DE, et al. Consolidative radiotherapy for limited metastatic non-small-cell lung cancer: a phase 2 randomized clinical trial. JAMA Oncol 2018;4:e173501.

41. Morris VK, Rashid A, Rodriguez-Bigas M, et al. Clinicopathologic features associated with human papillomavirus/p16 in patients with metastatic squamous cell carcinoma of the anal canal. Oncologist 2015;20:1247-1252.

42. Alfa-Wali M, Allen-Mersh T, Antoniou A, et al. Chemoradiotherapy for anal cancer in HIV patients causes prolonged CD4 cell count suppression. Ann Oncol 2012:23:141-147.

\section{See JNCCN.org for supplemental online content.}

